Ursprünge der Huachicolero-Bewegung zu beseitigen, werden öffentliche Investitionen noch intensiver in die bisherigen Cebiete mit Pipelines fließen. Die Kosten steigen. Obradors Blick auf die Wirtschaft bleibt letzten Endes an die extraktivistische Vision gebunden, die auch das Leben der Opfer von Tlahuelilpan forderte. Auch sie dachten, wie die Regierung, dass Reichtum durch Öl entstehe. Diese Einstellung kostet: Celd und Menschenleben. (Lomnitz 2019)

Dem ist hinzuzufügen, dass die geplanten Aktionen wie bei der Tragödie im Staat Hidalgo und zahlreichen ähnlichen Fällen in Puebla, Guanajuato und anderen Staaten, sowie das Aufeinandertreffen zwischen Menschen, Polizei und Militär und die nachgewiesene Komplizenschaft gewöhnlicher Anwohner mit Mafias ernstzunehmende Hindernisse für jede Politik zur Wiederherstellung der Staatsbürgerschaft darstellen: Es handelt sich um Strategien gegen den Staat. Die Wiederherstellung eines Gefühls der Öffentlichkeit und der Gemeinschaft ist umso anstrengender, wenn die Regierung selbst verlautet, dass $80 \%$ der Diebstahlfälle von Mitarbeitern des öffentlichen Unternehmens Pemex begangen wurden. Wie soll der Kampf gegen die Korruption und für eine gerechtere Nutzung der öffentlichen Güter, vom Öl bis hin zu den zuständigen Institutionen, angegangen werden?

\title{
Bürger ohne Staat
}

Das beschriebene, weitverbreitete Gefühl der Hilflosigkeit entsteht, wie wir sehen werden, nicht in einem Zeitraum von ein bis zwei Jahren. Seine internationale Dimension widerlegt Erklärungen, die versuchen, die Katastrophen mit der jeweiligen nationalen Geschichte zu begründen. Die Ereignisse müssen einzeln berücksichtigt werden, um nicht in ein und derselben grauen Masse der Prozesse unterzugehen. Man muss unterscheiden zwischen den White Anglo-Saxon Protestants (WASP), die für Trump stimmten, den brasilianischen Randgruppen, die Bolsonaro an die Macht gewählt haben, den Gelbwesten aus Frankreich und den mexikanischen Huachicoleros. Ihre nationalen Kontexte bringen unterschiedliche Interessenlagen mit sich. Auf der anderen Seite gehen ihre Gleichzeitigkeit, der Rückzug der Parteien und Gewerkschaften sowie die Nutzung informeller Netzwerke und der Mobiltelefone zur Durchsetzung und Kommunikation ihrer Forderungen über den lokalen $\mathrm{Zu-}$ sammenhang hinaus. Worin sind sie sich also ähnlich? 
Ich stelle die Hypothese auf, dass es in verschiedenen Ländern im Zuge der ökonomischen Krise und im Kontext der wirtschaftlichen und kommunikativen Globalisierung zu parallelen Zerwürfnissen zwischen den Eliten und breiten Teilen der Bevölkerung gekommen ist hinsichtlich der Frage, wie Gesellschaft gestaltet werden soll. Ein globales System, von dem wir dachten, es funktioniere, ist gerade dabei, zusammenzubrechen. Diese transnationale Entbürgerlichung verläuft auf mehreren Ebenen. Dafür werden in der Regel drei Gründe aufgeführt: a) die Elitisierung der Parteien und ihre Abkoppelung von ihrer sozialen Basis, b) die Kommodifizierung der Medien, und damit die Reduzierung ihrer gesellschaftlichen Funktion, und c) der Eindruck von Seiten der Bürger, dass ihre Proteste und Bedürfnisse nicht mehr von den öffentlichen Einrichtungen gehört und beantwortet werden. Bürger zu sein bedeutet heute nicht mehr das, was es früher bedeutete, oder was man sich darunter vor der Ära des globalen Kapitalismus vorstellte. Heute erleben wir ein anderes Verständnis von Politik: Staaten schließen abkommen mit Unternehmen. Soziale Grundrechte, die ursprünglich sinnstiftend für die moderne und demokratische Konzeption der Staatsbürgerschaft waren, werden heute flexibel geändert oder revidiert. Wendy Brown bezeichnet den Zusammenbruch dieser Idee von Demokratie als »Entdemokratisierung« (2005).

Die Auswirkungen von Fake News sind nur ein lauter Nebeneffekt der langjährigen Diskrepanzen zwischen Versprechen und Handeln. Technologische Neuerungen sind ein Grund, weshalb wir Durchschnittsbürger den Eindruck haben, in einem radikal neuen und modernen Zeitalter zu leben, in dem die Wissensproduktion und die Verbreitung von und der Zugriff auf Information revolutioniert worden sind. In diesem Buch wird argumentiert, warum die Entmündigung der Bürger ihre Vorgeschichte in früheren Prozessen hat. Wir haben es mit Brüchen und Ungleichmäßigkeiten zu tun, die, wie bereits erwähnt, nicht nur technologischer Natur sind, sondern auch als Folge der kognitiven Reorganisation des Kapitalismus zu bewerten sind (Lash 2007; Moulier 2007; Lins 2018). Es handelt sich um einen viel komplexeren Prozess als denjenigen, den die marxistische Tradition bloß als die Dissoziation zwischen Sprache und deren Anwendung im ideologischen Kontext kennt.

Die angeführten Veränderungen des Wissens und seiner digitalen Zirkulation sind allerdings nicht nur auf Technologien und auf die kapitalistische Wirtschaftsordnung, sondern auch auf die Umgestaltung des sozialen und kulturellen Sektors sowie des demokratischen Führungsstils zurückzuführen. Folglich ist eine Analyse erforderlich, die diese Mutationen und ihre Verflechtung im Zusammenhang betrachtet. Im Unterschied zu soziologischen 
Analysen und zur politischen Philosophie, die sich der Untersuchung des $\mathrm{Zu-}$ sammenbruchs der Demokratie als Legitimitäts- und Vertretungskrise der Parteien und der Steuerung von Unternehmen und Medien widmet, zeigen andere Betrachtungsweisen die Perspektive der Bürger auf. Sie befassen sich mit der Frage, wie diese über illegale oder informelle Wege nichtsdestotrotz ihre Ansprüche geltend machen, sie kommunizieren und sichtbar machen.

In diesem Buch soll untersucht werden, inwieweit das Ungleichgewicht in den Produktionsverhältnissen und der politischen Kultur der Regierungen, sowie in den Herausforderungen der privaten Akteure und den technologischen Möglichkeiten zu deren Ersetzung einen anderen Prozess des Wandels beleuchtet: den Bruch mit den kulturellen Grundlagen des Konzepts der Staatsbürgerschaft.

Die Macht der Medien und der digitalen Netzwerke führt Journalisten und Politologen dazu, die Theorie der Massenmanipulation durch diese Akteure wieder aufzugreifen. Dieser Ansatz wurde in den Kommunikationswissenschaften seit den 1970er-Jahren eigentlich als überholt eingestuft. Andere verweisen wiederum auf das Vordringen konservativer evangelikaler Kirchen, oder sie kritisieren die durch populistische Diskurse generierten Illusionen. Außerdem wird auf den Einfluss krimineller Organisationen und deren »Dienstleistungen« verwiesen, die zum Erhalt zahlreicher Sektoren beitragen. Diese vagen Hypothesen erklären kaum die Bandbreite der Erscheinungen, und sie liefern keine ausreichende Erklärung für die Strategien, die die Medien, die Kirche, die Politik und die kriminellen Kartelle anwenden. Deshalb muss nach anderen Erklärungsmöglichkeiten für diese Veränderungen in den Lebensverhältnissen, der Denkweise und der Unterstützung für hegemoniale Akteure gesucht werden. Die kulturelle Krise der Staatsbürgerschaft offenbart neue Pakte zwischen »dem gemeinen Volk« und den Führungspersonen aus Politik, Wirtschaft und Medien, seien sie illegal oder legal, und sie machen die Bandbreite an Überlebens- und Wohlstandsstrategien sichtbar, die unter dem Deckmantel des Neoliberalismus stehen.

Diese Diskrepanz zwischen Reden und Handeln und dem Wissen über die Funktionsweise unseres Landes oder der Welt und den effektiven Strategien, um sie verändern zu können, hat sich durch die COVID-Pandemie noch vergrößert. Was wir über die Verwendung persönlicher Daten zur Beeinflussung unseres Verhaltens und unserer Erkenntnis über den Anspruch auf unsere Rechte gelernt haben, hat für Unruhe in der politischen Führung gesorgt (vgl. der Wahlskandal unter dem Einfluss von Cambridge Analytica und andere Wahlmanipulationen). Mit dieser neuen Erkenntnis wachsen 
auch die Bewegungen des Feminismus, des Antirassismus, des Umweltschutzes sowie gegen die Prekarität in der jungen Generation. In den europäischen Parlamenten und im US-Kongress und bei den Straßenprotesten, die die oberen politischen Etagen scharf kritisieren, ist dies sichtbar geworden. Wenige Erfahrungen haben eine so große öffentliche Reichweite, wie die der feministischen Proteste und der Opposition gegen das historische Vermächtnis von Pinochet in der chilenischen Verfassung und in der Gesellschaft, oder wie die unkontrollierbaren Protestmärsche in den Städten: Sie fanden ihren Höhepunkt in den Demonstrationen am Internationalen Frauentag am 8. März 2020 und in der sinkenden Zustimmungsrate auf magere $6 \%$ für Präsident Piñera in der gleichen Woche. Er hatte die Quarantäne ausgenutzt, um die Bürger in ihren Häusern festzuhalten. Die überwältigende Mehrheit von $78 \%$, die die Pinochet-Verfassung am 25. Oktober 2020 mit deutlich größerer Wahlbeteiligung als bei früheren Wahlen abwählte, hat die Hegemonie der Rechten in Chile damit erschüttert.

Damit wurde deutlich, dass die Einschränkungen aus Angst vor dem Coronavirus kein Ende der anhaltenden Proteste in den sozialen Netzwerken bedeuten. In vielen Ländern haben die Demonstranten selbst angesichts der größten Gefährdung für die Gesundheit den öffentlichen Raum eingenommen. Das bekannteste Beispiel ist das der entrüsteten US-Bürger nach der Ermordung an George Floyd, deren Bewegung \#BlackLivesMatter in mehreren Ländern ein Echo fand, und die auf Proteste gegen andere Formen der Diskriminierung ausgeweitet wurde. Die verschiedenen Programmpunkte des physischen und virtuellen Protests vermischen sich mit Misstrauen gegenüber offiziellen Zahlen und politischen Maßnahmen angesichts der Pandemie. Sie zeigen die sozioökonomische Zersplitterung und die Komplexität der globalisierten Missstände, die reale Ängste und deren herbeigeführte Verschlimmerung nicht aufhebt. Selbst unter den Einschränkungen des öffentlichen Lebens, die uns die Pandemie auferlegt hat, sind wir Zeugen von Bewegungen zur Wiederherstellung der Staatsbürgerschaft geworden. Nicht nur die politisch motivierten, sondern auch »einfache" Bürger-Verbraucher sind den Regierungen einen Schritt voraus (und kompensieren deren fehlenden Handlungswillen), indem sie sich selbst organisieren. Bürger versorgen sich in der aktuellen Lage selbst, verteilen Lebensmittel oder bieten Pflege für Nachbarn an. Sie teilen Informationen schnell und mit globaler Reichweite. Wissenschaftliche Netzwerke und Journalistengruppen, die Informationen vergleichen und sie aufFakten prüfen, weiten ihre neuen Wissensmodelle und innovative Vorgehensweisen als Bürger aus.

Die Parteien und Gewerkschaften, die weltweit seit Jahrzehnten in Verruf geraten sind, sind in der aktuellen Lage jedoch stumm geblieben. Zwar gibt es Solidaritätsbewegungen (z.B. während der Erdbeben in Mexiko), die besonders intensiv und von kurzer Dauer sind. Es bleibt jedoch abzuwarten, welche solcher innovativen Initiativen im Kontext einer Post-Pandemie eine sinnvolle Vertiefung anbieten würden. Geht es um 
die Straßenproteste, die in den sozialen Netzwerken zunächst an Stärke gewinnen und Unternehmen dazu zwingen, Falschmeldungen aus dem Verkehr zu ziehen oder gegen die Bürger gerichteten Regierungen keine Daten zu verkaufen, so stellt sich die Frage, ob sie sich durch das wachsende Aufkommen von Online-Netzwerken nochmal bestätigen werden: Nachbarschaftsnetzwerke, Kollegengruppen, Freundesgruppen, Migrantencommunitys, nonkonforme Experten, und viele andere? Das Bürgersein lässt sich nicht darauf reduzieren, was die Algorithmen mit uns oder mit den großen Unternehmen anstellen.

\section{Die einvernehmliche Unterordnung}

In der jüngsten Phase des Zusammenbruchs der politischen Parteien in Brasilien wurde der Versuch unternommen, Konzepte aus den Sozialwissenschaften neu zu schreiben. Statt das scheinbar irrationale Wählerverhalten zu beurteilen, fragt man sich nach alternativen Wegen, dieses zu verstehen. Der brasilianische Soziologe Carlos Eduardo Martins beschrieb den durch den Sturz Dilma Rousseffs ausgelösten Ausnahmezustand, der im Wahlsieg von Bolsonaro gipfelte, als »faschistisch-liberal«. Er argumentiert, dass diese Verschmelzung zweier scheinbar gegensätzlicher politischer Strömungen sich aktuell dadurch auszeichnet, dass zwar Grundsätze der liberalen Demokratie unter Beibehaltung ihrer formalen Aspekte erhalten bleiben. Die autokratische Machtstruktur, die den politischen Wettbewerb einschränkt und die freie Ausübung der Souveränität oder Proteste durch das Volk verhindert, dringe jedoch weiter vor. Seit dem Wahlkampf in Brasilien, so Martins, beobachten wir den Einsatz begrenzter, aber strategischer Maßnahmen der Gewalt, der Willkür und des Terrors. Sie werden eingesetzt, um Arbeitnehmer- oder Menschenrechtsbewegungen sowie die Opfer des in den Drogenhandel verwickelten Staats zum Schweigen zu bringen. Unterdessen werden neue Bündnisse eingegangen, und zwar zwischen regierungsunfähigen Eliten und transnationalen Megakonzernen, sowie Teilen der Mittel- und Unterschicht, die von der Rezession betroffen sind. Ähnliche Prozesse treten in den letzten Jahren in Argentinien, Mexiko und einigen zentralamerikanischen Ländern auf, wenn auch in kleinerem Maßstab.

Diese Erklärungen stützen die gängigen Argumente bezüglich der Neuformierung der Machtverhältnisse durch die Rechten. Es gibt immer noch keine vollständige Erklärung für die grundlegenden Ursachen dieser massenhaften Zustimmung oder für die Umwandlung der Arbeiterklasse und ande- 